\title{
One, No One, and One Hundred Thousand: The Paradigm of the $Z-R$ Relationship
}

\author{
MASSIMILIANO IGNACCOLO \\ SAS Institute, Cary, North Carolina \\ CARlo De Michele \\ Politecnico di Milano, Milano, Italy
}

(Manuscript received 11 August 2019, in final form 8 February 2020)

\begin{abstract}
The $Z-R$ relationship is a scaling-law formulation, $Z=A R^{b}$, connecting the radar reflectivity $Z$ to the rain rate $R$. However, more than $100 Z-R$ relationships, with different values of the parameters, have been reported in literature. This abundance of relationships is in itself a strong indication that no one "physical" relationship exists, a state of affairs that we find similar to that of the protagonist of Luigi Pirandello's novel One, No One and One Hundred Thousand. Nevertheless the "elevation" of a simple linear fit in the $(\log R$, $\log Z$ ) space to the role of "scaling law" is such a widespread tenet in literature that it eclipses the simple realization that the abundance of different intercepts and slopes reflects the inhomogeneous nature of rain, and, in ultimate analysis, the statistical variability existing between the number of drops and drop size distribution. Here, we "eliminate" the contribution of the number of drops by rescaling both reflectivity and rainfall rate to per unit drop variables, $(Z, R) \rightarrow(z, r)$, so that the remaining variability is due only to the variability of the drop size distribution. We use a worldwide database of disdrometer data to show that for the rescaled variables $(z, r)$ only "one," albeit approximate, scaling law exists.
\end{abstract}

\section{Introduction}

Since the end of Second World War, radar measurements have become of paramount importance in meteorology and hydrology, because they represent the principal way of investigating the spatial variability of the rain phenomenon $\left(\sim 1 \mathrm{~km}^{2}\right.$ as typical spatial resolution) (Thorndahl et al. 2017) as opposed to the "point" ( $\sim 50-100 \mathrm{~cm}^{2}$ as sampling area) information offered by, for example, rain gauges and disdrometers (Atlas 1990). The conversion between the radar reflectivity factor $Z$ and the rain rate $R$ represents the main issue in order to retrieve rain measurements from weather radar signals.

In the last 70 years, this conversion has been mainly operated through a power-law $(Z-R)$ relationship

$$
Z=A R^{b},
$$

with $Z$ in units of $\mathrm{mm}^{6} \mathrm{~m}^{-3}, R$ in units of $\mathrm{mm} \mathrm{h}^{-1}$, and $A$ and $b$ are two parameters evaluated from a linear regression between observed values of $\log Z$ and $\log R$.

Corresponding author: Massimiliano Ignaccolo, massimiliano. ignaccolo@sas.com
The relation proposed by Marshall et al. (1955) $(Z=$ $\left.200 R^{1.6}\right)$ is considered as a default relationship in some countries (Raghavan 2013), and represents one of the most used $Z-R$ relationships. In literature, more than $100 Z-R$ relationships are available, with the following range for the parameters: $A \in[30,1000], b \in[0.8,2]$ (see, e.g., Stout and Mueller 1968; Battan 1973, Tables 7.1-7.2; Atlas 1990; Uijlenhoet 2001; Rosenfeld and Ulbrich 2003; Raghavan 2013, Table 7.2; Orellana-Alvear et al. 2017; Rauber and Nesbitt 2018, chapter 13). Considering the variability of $A$ and $b$ parameters available in literature, Stout and Mueller (1968) investigated the differences in rainfall rate at the same reflectivity using different $Z-R$ power-law relationships: they report differences up to $500 \%$. The great spectrum of possible values reported for the parameters $(A, b)$ is due to the differences in geographical location, the type of rain (stratiform, convective, and orographic), different epochs within a single rain event, the mixing of different precipitation phases, and also uncertainty and errors in measurements (Tokay et al. 2001; Licznar and Krajewski 2016).

Reflectivity-rainfall rate relationships were developed for the different types of rain, with the hope of improving the estimation of rainfall rate from the reflectivity. 
However, even if one focuses the attention to the differences between stratiform and convective rain, contrasting results are obtained: Yuter and Houze (1997) found that the parameter $A$ for convective rains was higher than the one in stratiform rains, while Tokay and Short (1996) and Atlas et al. (1999) found the opposite. Ochou et al. (2011) found that both the two situations were obtained in their analysis, suggesting that the parameter $A$ for convective rains can be higher or lower the one in stratiform rains.

In their seminal work Marshall and Palmer (1948) used an exponential form for the probability distribution of rain drop diameter, with a decaying constant $L$ depending on the rain rate: $L=41 R^{-0.21} \mathrm{~cm}^{-1}$. This choice leads to a $Z-R$ relationship with $A=296$, and $b=1.47$. Atlas and Chmela (1957) found a linear $Z-R$ relationship ( $b=1$ ), where the coefficient $A$ is a function of the drop size distribution. List (1988) suggested that linear $Z-R$ relationship applies only to equilibrium drop size distributions. He proposed the relation $Z=742 R$, for steady tropical rain, with $A=742$, as universal constant. Jameson and Kostinski $(2001,2002)$ argued 1) that a linear $Z-R$ relationship is possible only for statistically homogeneous rain and 2) that the $Z-R$ relationship is not "physical" but statistical in nature because of the inhomogeneous nature of rain, hence the proliferation of power-law $Z-R$ relationships. Steiner et al. (2004) identified three particular conditions in which a physical $Z-R$ relationship occurs based on exponential, gamma, and monodisperse raindrop size distributions. However, the existence in nature of the conditions highlighted by Steiner et al. (2004) remains to be evaluated.

\section{Results}

In this work, we show that an "approximate" universal law exists upon rescaling the variables by the number of drops:

$$
z=C \zeta_{p} r^{2}
$$

where $z$ is the reflectivity per unit drop, $r$ the rainfall rate per unit drop, $C$ is a constant [Eq. (B20)], and $\zeta_{p}$ [Eq. (B21)] is a "form" factor depending on the shape of the drop size distribution and the relation between rain drop velocity and drop diameter. The above equation is an exact mathematical relationship (no particular assumption on the functional form of the drop size distribution is necessary to obtain it, see appendix B) but "physically" it represents an approximate scaling law. This is so because, while the form factor is not always constant, its variability is "small" (say, in the range $[0.5,4]$; see Figs. 1 and 4).

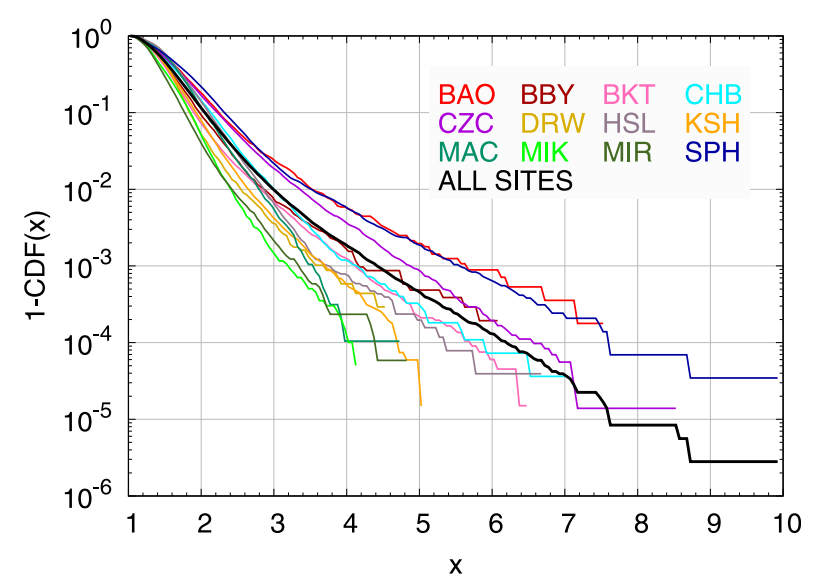

FIG. 1. Comparison of values of the form factor $\zeta_{p}$ at 12 different sites by use of RD80 Joss-Waldvogel disdrometers. The colored lines indicate the survival function $S\left(\zeta_{p}\right)$ (the observed frequency of a form factor value $\geq \zeta_{p}$ ) at the different sites. The "ALL SITES" line (black) reports the survival functions estimated by merging all datasets.

To prove this point, we operate as follows. First, we consider data from 12 RD80 Joss-Waldvogel disdrometers located in different sites on Earth's surface (see appendix A). Using the RD80 data (a total of 227823 one-minute counts), we show how the form factor $\zeta_{p}$ of Eq. (2) is small independently from site and synoptic condition. Then, we consider two additional sites: one with a 2DVD disdrometer (1699 one-minute counts), and one with a Thies optical disdrometer (18911 one-minute counts). With these additional datasets, we show that the form factor $\zeta_{p}$ is small independently from the instrumentation used to "measure" the rainfall phenomenon. Finally, while the 2DVD and Thies disdrometers directly report the drop velocity, no velocity information is available for the RD80 disdrometer. In this case an analytical functional form $v(D)$ is used to calculate the velocity of a rain drop: we use the RD80 dataset to show that the form factor $\zeta_{p}$ is small, not depending on the choices of $v(D)$ available in literature.

For our numerical estimations, we use the indirect method to calculate both $(Z, z)$ and $(R, r)$ from the rain drop size distribution, and then estimate the form factor $\zeta_{p}$. All the details about 1) derivation and numerical estimation of Eq. (2) and 2) datasets and their processing are covered in great extent in appendixes A and B. This is so, in order to focus the attention of the reader on the "generality" of the result as the great majority of the material presented in the appendixes is already abundantly covered in literature.

\section{a. Independence from site and synoptic condition}

We use 12 RD80 Joss-Waldvogel datasets and assume the rain drop velocity to be $v(D)=3.78 D^{0.67}$, in meters 


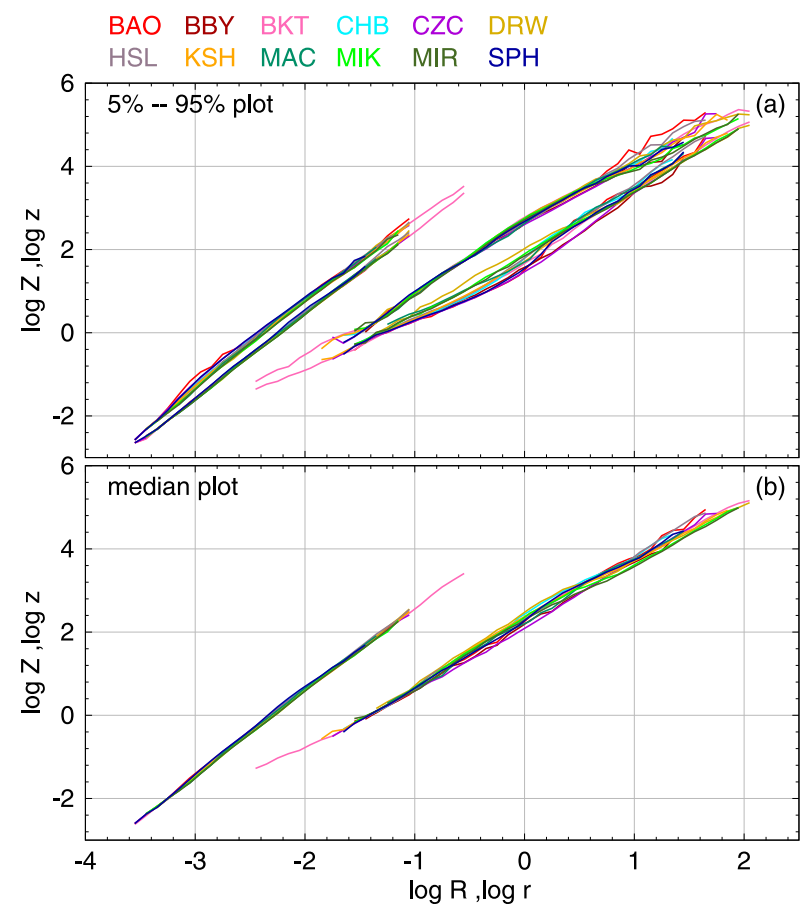

FIG. 2. (a) The $5 \%$ and $95 \%$ percentile curves for $\log Z(\log z)$ given a fixed value of $\log R(\log r)$ for all 12 sites considered. (b) The median curves for $\log Z(\log z)$ given a fixed value of $\log R(\log r)$ for all 12 sites considered. The bin size for $\log R(\log r)$ used to make the plot is 0.1: only bins with at least 10 observations in it were considered.

per second $\left(\mathrm{m} \mathrm{s}^{-1}\right)$ and $D$ in millimeters $(\mathrm{mm})$, as in (Atlas and Ulbrich 1977). The black line in Fig. 1 shows how for $90 \%$ of the 1-min spectra considered the form factor $\zeta_{p}$ is a number between 1 and 2, with $99 \%$ of the 1-min spectra with a form factor between 1 and 3. If we consider the form factor for each one of the 12 sites (different colors in Fig. 1), we see that 8 sites have less variability than the overall sample (black line), with the "worst performing" site being BAO (red curve) where $99 \%$ of 1-min spectra considered have a form factor between 1 and 3.5.

To further support the evidences depicted in Fig. 1, we consider, at each site, the "spreading" of 1-min spectra in the $(\log R, \log Z)$ and $(\log r, \log z)$ planes. To quantify the spreading, we fix a value of $\log R(\log r)$ and calculate the $5 \%$ and $95 \%$ percentile of the values of $\log Z(\log z)$ associated with it. The resulting $5 \%$ and $95 \%$ percentile curves are plotted in Fig. 2a. We see how the spreading for the rescaled couple $(\log r, \log z)$ is smaller than the one of the couple $(\log R, \log Z)$. Moreover, in Fig. 2b we see how the median curves are better aligned for the $(\log r$, $\log z)$ couple rather than for the $(\log R, \log Z)$ couple.

Finally, we show in Fig. 3 how the Eq. (2) is not dependent on the particular type of rain considered.

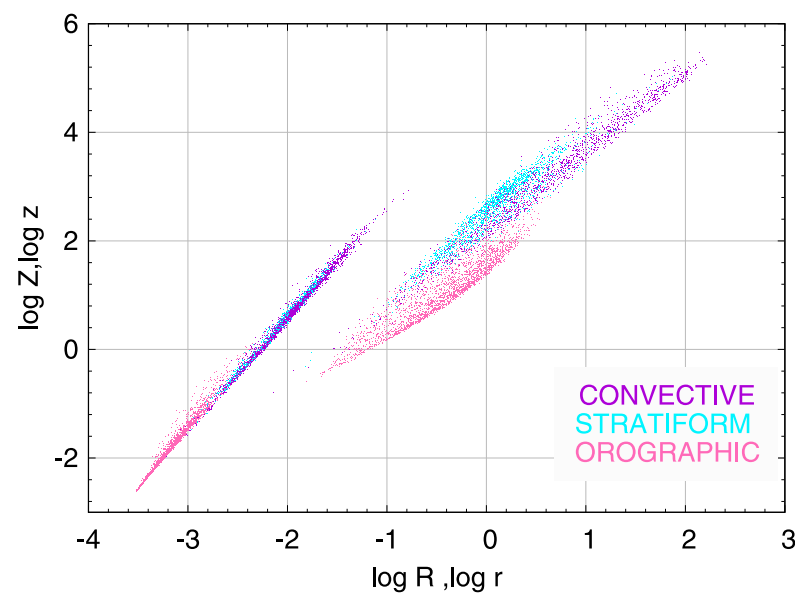

FIG. 3. Shown are $\log Z$ vs $\log R$, and $\log z$ vs $\log r$ distinguishing convective by stratiform for Darwin and stratiform by orographic for Cazadero. Each dot indicates a 1-min disdrometer record.

The distinction between stratiform and convective, made according to the presence or absence of bright band, regards the Darwin dataset (DRW) as in Williams (2009). Similarly, the distinction between stratiform and orographic made according to the presence or absence of bright band regards the Cazadero dataset (CZC) as in Martner et al. (2008): additional details can be found also in Ignaccolo and De Michele (2010, 2012a,b).

\section{b. Independence from rain drop velocity functional form and type of instrument}

We now consider the unified RD80 datasets (obtained by merging all the 12 single site RD 80 datasets) together with the 2DVDV and Thies datasets.

The gray line in Fig. 4 shows the survival probability for the form factor $\zeta_{p}$ relative to the unified RD80 dataset when we use for the rain drop velocity the relation $v(D)=9.65-10.3 e^{-0.6 D}$, in meters per second and $D$ in millimeters, proposed in Atlas et al. (1973): a popular choice in literature. Compared to the case of $v(D)=3.78 D^{0.67}$ (Atlas and Ulbrich 1977) reported as a black line (same as black line in Fig. 1), the form factor $\zeta_{p}$ is contained in a "smaller" range.

The 2DVD reports the speed of each single drop, while the Thies disdrometer classifies drops in joint (diameter, velocity) classes. Therefore, it is not necessary, for these instruments, to assume any functional form for the velocity of a rain drop in order to calculate the reflectivities $Z$ and $z$. We see in Fig. 4 that $99 \%$ of the 1-min counts of the 2DVD dataset (green line) has a form factor in the range [0.5, 4], while the range becomes $[1,8]$ for the Thies dataset. We think that the larger range observed for the Thies dataset is partially due to the Thies coarser measurement 


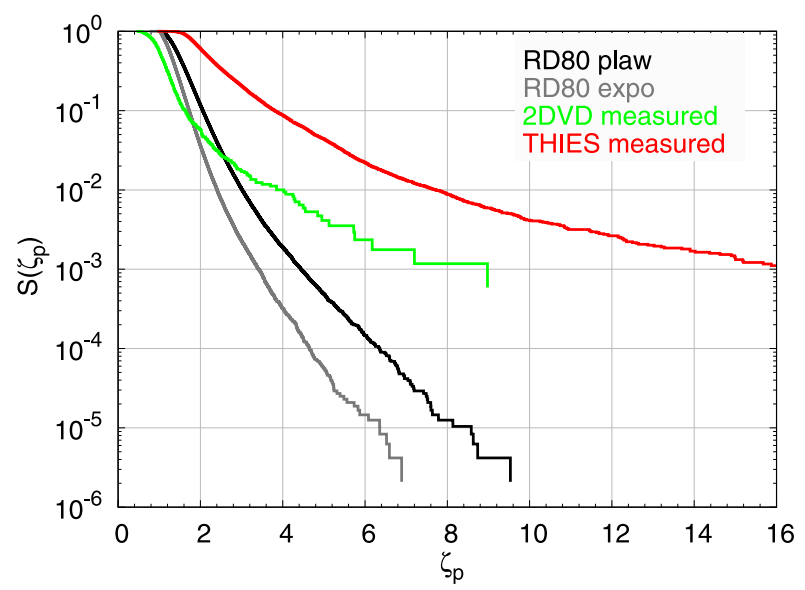

FIG. 4. Comparison of values of the form factor $\zeta_{p}$ for different instruments. The colored lines indicate the survival function $S\left(\zeta_{p}\right)$ (the observed frequency of a form factor value $\geq \zeta_{p}$ ) for RD80 (black and gray), 2DVD (green), and Thies (red) datasets.

of the rain drop speed with respect to the 2DVD disdrometer.

Overall Figs. 1-4 corroborate the idea that the "smallness" of the form factor $\zeta_{p}$ is a general property of the rainfall phenomenon.

\section{Discussion and conclusions}

Distinguishing between scaling laws and empirical fits is an important challenge in Hydrology. There is no scaling law between $Z$ and $R$ as Eq. (1) has not the "quality" of a law such as, for example, $E=m c^{2}$ has. This latter equation states that energy is proportional to mass (the speed of light squared being the proportionality constant and the scaling exponent of the mass being 1). There is only one scaling exponent (not a myriad of them) and one constant factor (not a myriad of them). The law $E=m c^{2}$ is a bone fide scaling law, while Eq. (1) is not.

As pointed out in Jameson and Kostinski (2001), one cannot consider Eq. (1) as a physical law "unless one can explain precisely and physically how $N$ determines $p(D)$ or, conversely, how $p(D)$ determines $N$ ": we could not agree more. It is how the relationship between $N$ and $p(D)$ affects the connection between $Z$ and $R$ that we explore in this work. We "isolated" the contribution of the variability of the number of drops $N$ by rescaling $(Z, R)$ to "per unit drop" variables $(z, r)$ and we found that, in spite of the great variability of $p(D)$, approximately "One" relationship exists [Eq. (2)] for the rescaled variables. Alternatively, our results can be stated as follows: "the fact that to a given $p(D)$ is associated a large range of possible values of $N$ is what explains most of the variability observed in the $\log Z-\log R$ plot, while the contribution of different shapes of $p(D)$ is quite modest."

It is our opinion that given the results presented here, a possible course of action to improve rainfall rate retrieval from radar observations would be along the following lines.

0) Stop talking about "scaling law" for the relationship among rainfall bulk variables. The word "law" gives the false impression the Eq. (1) is a physical property of the rainfall phenomenon: it is not.

1) Investigate the relationship between $N$ and $p(D)$ without assuming any particular functional form for $p(D)$ [the analyses presented by Ignaccolo and De Michele (2012a,b) goes in this direction]. Also the Gamma distribution is not the proper functional form for $p(D)$ : it has difficulties to pass nonparametric goodness-of-fit tests (Ignaccolo and De Michele 2014), presents worst performances compared to other distributions (Cugerone and De Michele 2015; D'Adderio et al. 2016).

2) In lieu of steps 0 and 1 , stop fitting regression lines into $\log -\log$ plots of $Z$ versus $R$, we have been doing it since the inception of radar meteorology: there are more sophisticated statistical tools easily accessible to any present day personal computer. For example, one could build machine learning models connecting $Z$ to $N$ and the statistical moments of $p(D)$ and therefore to $R$ : note $N$ together with mean, standard deviation, and skewness of $p(D)$ uniquely determine $R$ (Ignaccolo and De Michele 2014).

3) Take step 2 one step further: for example, add "meteorological" variables to the machine learning models to obtain more sophisticated $Z$ versus $R$ relationships.

Of course, steps $0-3$ reflect the particular "inclinations" and thoughts of the authors and are by no means "diktats." Moreover, it is not our intention to belittle the value of years of hard work from the community resulting in much progress in the field of remote sensing estimation of rainfall. We are, based on the results of this manuscript, mostly advocating for a new approach and a different tenet: one that is more closely founded on observed "universal" property of the rainfall phenomenon, one that mirrors more closely the present day methodology of data science, one that, in our opinion, will lead to practical progresses in radar hydrometeorology.

Acknowledgments. The authors thank the editor, Dr. Wade T. Crow, and three anonymous reviewers for the comments and suggestions during the review process. 
TABLE A1. List of the sites where disdrometer data are collected with code, latitude, longitude, altitude, type of disdrometer (JWD is Joss-Waldvogel disdrometer, 2DVD is two-dimensional video disdrometer, and TD is Thies disdrometer), number of minutes, and total number of drops in the dataset.

\begin{tabular}{|c|c|c|c|c|c|c|c|}
\hline Site & Code & Lat $\left(^{\circ}\right)$ & Lon $\left(^{\circ}\right)$ & Alt (m) & Type & No. of minutes & No. of drops \\
\hline Eire $(\mathrm{CO})$, United States & $\mathrm{BAO}$ & $40.05^{\circ} \mathrm{N}$ & $105.00^{\circ} \mathrm{W}$ & 1577 & JWD & 6016 & 2349280 \\
\hline Bodega Bay (CA), United States & BBY & $38.20^{\circ} \mathrm{N}$ & $123.00^{\circ} \mathrm{W}$ & 12 & JWD & 10804 & 5389240 \\
\hline Bukit Koto Tabang, Indonesia & BKT & $0.12^{\circ} \mathrm{S}$ & $100.19^{\circ} \mathrm{E}$ & 864 & JWD & 68389 & 25109376 \\
\hline Chilbolton, United Kingdom & $\mathrm{CHB}$ & $51.14^{\circ} \mathrm{N}$ & $1.43^{\circ} \mathrm{W}$ & 82 & JWD & 29122 & 7015480 \\
\hline Cazadero (CA), United States & $\mathrm{CZC}$ & $38.61^{\circ} \mathrm{N}$ & $123.22^{\circ} \mathrm{W}$ & 475 & JWD & 76137 & 44252384 \\
\hline Darwin, Australia & DRW & $12.45^{\circ} \mathrm{S}$ & $130.83^{\circ} \mathrm{E}$ & 12 & JWD & 6863 & 2753037 \\
\hline Hassel, Germany & HSL & $51.51^{\circ} \mathrm{N}$ & $7.1^{\circ} \mathrm{E}$ & 60 & JWD & 26402 & 7072649 \\
\hline Kashima, Japan & $\mathrm{KSH}$ & $35.95^{\circ} \mathrm{S}$ & $140.65^{\circ} \mathrm{E}$ & 45 & JWD & 68570 & 19752935 \\
\hline Macunaga, Italy & MAC & $45.97^{\circ} \mathrm{N}$ & $7.96^{\circ} \mathrm{E}$ & 1300 & JWD & 9956 & 3275264 \\
\hline Kwajalein Atoll, Marshall Islands & MIK & $8.71^{\circ} \mathrm{N}$ & $167.73^{\circ} \mathrm{E}$ & 7 & JWD & 20170 & 7594915 \\
\hline Roi Namur, Marshall Islands & MIR & $9.39^{\circ} \mathrm{N}$ & $167.47^{\circ} \mathrm{E}$ & 5 & JWD & 17518 & 7328949 \\
\hline Sparsholt, United Kingdom & SPH & $51.14^{\circ} \mathrm{N}$ & $1.43^{\circ} \mathrm{W}$ & 118 & JWD & 31063 & 11167510 \\
\hline Southern Great Plains (OK), United States & OKL & $36.60^{\circ} \mathrm{N}$ & $97.49^{\circ} \mathrm{W}$ & 314 & 2DVD & 1699 & 738821 \\
\hline Milano, Italy & MLN & $45.28^{\circ} \mathrm{N}$ & $9.23^{\circ} \mathrm{E}$ & 123 & $\mathrm{TD}$ & 18911 & 8817189 \\
\hline
\end{tabular}

\section{APPENDIX A}

\section{Data}

We use data from 12 RD80 Joss-Waldvogel (JWD) impact disdrometers, one two-dimensional video disdrometer (2DVD), and one Thies (TD) optical disdrometer. All disdrometers store data at 1-min time resolution, even if the 2DVD has a time resolution of $1 \mathrm{~ms}$. Table A1 reports the list of locations, with a threeletter site code, latitude, longitude, altitude, and the type of disdrometer. Joss-Waldvogel disdrometers register the diameter class (diameter range) to which a drop belongs. The Thies disdrometer collects the diameter class (diameter range) to which a drop belongs and also its velocity providing the velocity class. The 2DVD provides for each drop its diameter and velocity. For Joss-Waldvogel and Thies disdrometers, we assumed a uniform distribution of the drop diameter inside each diameter class: disdrometric quantization (Ignaccolo et al. 2009; Marzuki et al. 2010).

Data were processed as follows. We considered, for each database, only minutes for which the drop count is $\geq 60$ (drop arrival rate $\sim 1 \mathrm{~s}^{-1}$ ): this threshold (Ignaccolo et al. 2009) separates phases of active precipitation from phases that are quiescent, and also serve as a rule of thumb threshold for obtaining "statistically" meaningful 1-min spectra. For minutes with a drop count larger than 60 , active minutes, we then consider only "contiguous" counts. For example, a disdrometer reading of $[175,226,158,93,83,128,139,44,17,0,0,1$, $0,0,0,0,0,0,0,0]$ is converted in to $[175,226,158,93$, $83,128,139,44,17,0,0,0,0,0,0,0,0,0,0,0]$ by dropping the noncontiguous count on the eleventh diameter class whether or not it is a legit count or an artifact of the instrument: we will rather risk underestimating than overestimating the moments of $p(D)$. This "cleaning" procedure does not impact any of the results presented in this work as demonstrated in detail in the "Dealing with outliers" section in the appendix of Ignaccolo and De Michele (2014). Hereby, for brevity, we briefly summarize the "impact" of the noncontiguous count removals. One-minute spectra with noncontiguous counts are $\simeq 15 \%$ of the active minutes considered, but the procedure of removing drops in noncontiguous counts only results in $\simeq 1 \%-2 \%$ loss of drops. Typically, only one or at most two drops are removed, with the probability, at any given site, of removing, from a 1-min spectra, more than $10 \%$ of drops $\$ 1 \%-2 \%$.

The 2DVD dataset presents outliers in the velocity value of some drops: observed velocity values beyond the terminal velocity. This problem was highlighted by Kruger and Krajewski (2002). For, the detection and removal of outliers we used the procedure described by Kruger and Krajewski (2002). An outlier is defined if a drop has a velocity outside the range $\left|v_{\text {measured }}-v(D)\right|<0.4 v(D)$, where $v(D)$ is given by Eq. (B3) (Atlas et al. 1973).

The distinction between stratiform and convective, made according to the presence or absence of bright band, regards the Darwin dataset as reported in Williams (2009). Similarly, the distinction between stratiform and orographic made according to the presence or absence of bright band regards the Cazadero dataset as reported in Martner et al. (2008): further details can be found in Ignaccolo and De Michele (2010, 2012a,b).

Table A1 reports the number of minutes and drops considered in the analysis, after datasets are processed. 


\section{APPENDIX B}

\section{Methods}

\section{a. Statistical description of rainfall}

The statistical variability of the rain drop diameter is usually described by means of the concentration per unit volume and diameter $\mathscr{N}(D)=N_{V} f(D): N_{V}$ is the number of drops per cubic meter, and the probability density $f(D)$ is the "volume" diameter distribution. However, with the exception of some airborne observations (Yuter and Houze 1997), drop diameters are measured near the surface by disdrometers. These instruments report, at fixed interval of times $T$ (usually $1 \mathrm{~min}$ ), the number of drops $N$ fallen through the sampling area and the diameter of each drop, so that one can estimate the probability density $p(D)$ : the "flux" diameter distribution. The volume and the flux description of the rainfall are equivalent. One can switch between the two as follows (Uijlenhoet 2001; Ignaccolo and De Michele 2014):

$$
\mathscr{N}(D)=N_{V} f(D)=\frac{N}{A_{m} T v(D)} p(D) .
$$

In the above equation, $A_{m}$ is the sampling area of the instrument expressed in square meters, $T$ is the sampling time interval in seconds, and $v(D)$, in meters per second, is the velocity of a drop of diameter $D$. Hereby, we adopt the flux description.

The most common functional dependence for the velocity $v(D)$ adopted in the literature (Atlas and Ulbrich 1977) is

$$
v(D)=3.78 D^{0.67},
$$

with $v(D)$ in meters per second and $D$ in millimeters. This dependence allows for analytical expression for the moments of both volume and flux representation (see next subsection). A functional dependence that is considered (Atlas et al. 1973) to fit better observed rain drop velocities is

$$
v(D)=9.65-10.3 e^{-0.6 D},
$$

again with $v(D)$ in meters per second and $D$ in millimeters.

\section{b. Rainfall bulk variables and diameter distributions moments}

Rainfall bulk variables such as the reflectivity $Z$, rainfall rate $R$, and number concentration per cubic meter $N_{V}$ are related to the moments of either the flux or the volume drop diameter probability density. In particular, using Eq. (B1), we can write

$$
\left\{\begin{aligned}
R & =\frac{6 \pi 10^{-4}}{A_{m} T} N \int D^{3} p(D) d D=\frac{6 \pi 10^{-4}}{A_{m} T} N M_{3} \\
Z & =N_{V} \int D^{6} f(D) d D=\frac{1}{A_{m} T} N \int \frac{1}{v(D)} D^{6} p(D) d D=\frac{1}{A_{m} T} N M_{6, v} \\
N_{V} & =N_{V} \int f(D) d D=\frac{1}{A_{m} T} N \int \frac{1}{v(D)} p(D) d D=\frac{1}{A_{m} T} N M_{0, v}
\end{aligned}\right.
$$

The symbol $M_{\alpha}\left(M_{\alpha, v}\right)$ indicates the $\alpha$ th moment $[\alpha$ th moment weighted by $1 / v(D)]$ of the flux probability density $p(D)$. If the velocity $v(D)$ has the power-law functional form of Eq. (B2)

$$
M_{\alpha, v}=(1 / 3.78) \times M_{\alpha-0.67} .
$$

It is common in the literature (Ulbrich 1983; Kliche et al. 2008) to assume a gamma functional form for the volume drop diameter probability density $f(D)$. This functional choice has poor statistical support (Ignaccolo and De Michele 2014), and it is mostly adopted because in conjunction with the power-law dependence for the rain drop velocity [Eq. (B2)] "one has an analytical formula for the moments of any order." In the following, we do not assume any particular functional form for $p(D)$. Similarly, without loss of generality, we write the $\alpha$ th moment $M_{\alpha}$, and the $\alpha$ th moment weighted by $1 / v(D)$ as

$$
\left\{\begin{array}{c}
M_{\alpha}=\int D^{\alpha} p(D) d D=\eta(\alpha) \Gamma(\alpha+1) \mu^{\alpha} \\
M_{\alpha, v}=\int \frac{1}{v(D)} D^{\alpha} p(D) d D=\eta_{v}(\alpha) \frac{1}{3.78} \Gamma(\alpha+1-0.67) \mu^{\alpha-0.67}
\end{array}\right.
$$


where $\Gamma($.$) if the gamma function. The factor \eta(\alpha)$ indicates the departure of $p(D)$ from the exponential form and $\mu$ is the mean drop diameter $\left[\mu=\int D p(D) d D\right]: \eta(\alpha)=1$ if $p(D)$ is the exponential distribution. Similarly, when considering moments weighted by $1 / v(D)$, we also consider the departure from the power-law functional form of Eq. (B2): $\eta_{v}(\alpha)=1$ if $p(D)$ is the exponential distribution and $v(D)=3.78 D^{0.67}$.

\section{c. Numerical estimation of drop size distribution moments}

Disdrometers such as the Joss-Waldvogel RD80 report only counts per drop diameter classes and not the diameter of each drop. In this case, the flux probability density $p(D)$ as estimated from disdrometer counts is a step function with constant value inside the boundaries of each disdrometer class. Thus, the $\alpha$ th moment $M_{\alpha}$, the first formula in Eq. (B6), is estimated as

$$
M_{\alpha}=\sum_{j} p_{j}(D) \int_{D_{j, L}}^{D_{j, R}} D^{\alpha} d D=\sum_{j} \frac{n_{j}}{N \Delta_{j}} \times \frac{D_{j, R}^{\alpha+1}-D_{j, L}^{\alpha+1}}{\alpha+1},
$$

where the sum is taken over the diameter classes, index $j$. The symbol $p_{j}$ indicates the value of $p(D)$ inside the $j$ th diameter class and $N$ the total number of drops observed in the unit time interval, while $n_{j}, \Delta_{j}, D_{j, L}$, and $D_{j, R}$ are the drop count, width, left limit, and right limit of the $j$ th diameter class, respectively. The $\alpha$ th moment weighted by $1 / v(D) M_{\alpha, v}$, the second formula in Eq. (B6), is estimated using Eqs. (B5) and (B7) when $v(D)$ has the power-law form of Eq. (B2), and the following expression in the remaining cases:

$$
\begin{aligned}
M_{\alpha, v} & =\sum_{j} p_{j}(D) \int_{D_{j, L}}^{D_{j, R}} \frac{1}{v(D)} D^{\alpha} d D \\
& =\sum_{j}\left[\frac{n_{j}}{N \Delta_{j}} \times \sum_{k=1}^{K} \frac{D_{j k}^{\alpha}}{v\left(D_{j k}\right)} I_{j}\right] .
\end{aligned}
$$

In the above formula the $j$ th diameter class range is divided in $K$ equal intervals of length $I_{j}=\Delta_{j} / K$. For large enough $K$ (we adopt $K=25$ ) the function $D^{\alpha} / v(D)$ can be considered constant inside each interval $I_{j}$, and the integral approximated by a sum where $D_{j k}$ is the middle value of the $k$ th interval in which the $j$ th disdrometer class is divided.

A Thies disdrometer divides drop diameters in 22 classes and the drop velocities in 20 classes for a combined total of 440 classes (Frasson et al. 2011). Thus, a Thies drop count is indexed as $n_{j k}$ : the number of drops in the $j$ th diameter class having a velocity in $k$ th velocity class. For the moments $M_{\alpha}$, since they are not weighted by the drop velocity, one can aggregate the drop count through all the velocity classes and produce only drop counts at the size class level: $n_{j}=\sum_{K} n_{j k}$. After this step the $\alpha$ th moment $M_{\alpha}$ can be calculated via Eq. (B7). The estimation of the $\alpha$ th moment weighted by $1 / v(D) M_{\alpha, v}$ is done using

$$
\begin{aligned}
M_{\alpha, v} & =\sum_{j, k} p_{j k}(D) \int_{D_{j, L}}^{D_{j, R}} \frac{1}{v(D)} D^{\alpha} d D \\
& =\sum_{j, k}\left(\frac{n_{j k}}{N \Delta_{j}} \times \frac{1}{v_{j k}} \times \frac{D_{j, R}^{\alpha+1}-D_{j, L}^{\alpha+1}}{\alpha+1}\right),
\end{aligned}
$$

where $v_{j k}$ is the central values of the $k$ th velocity class (that is to say that all the drops in the in the $k$ th velocity class are considered to have the same velocity $v_{j k}$ ).

A 2DVD disdrometer reports the diameter (equivalent diameter as drops are not perfect spheres) and the velocity of each single drop. Therefore, given a time interval, the flux probability density of drop diameter $p(D)$ is the sum of several Dirac delta functions

$$
p(D)=\frac{1}{N} \sum_{n=1}^{N} \delta\left(D-D_{n}\right)
$$

where $N$ is the number of drops inside the time interval. As a consequence

$$
\left\{\begin{array}{c}
M_{\alpha}=\frac{1}{N} \sum_{n=1}^{N} D_{n}^{\alpha} \\
M_{\alpha, v}=\frac{1}{N_{n}} \sum_{n=1}^{N} \frac{1}{v_{n}} D_{n}^{\alpha}
\end{array}\right.
$$

where $v_{n}$ is the velocity reported by the instrument for the $n$th drop in the time interval considered.

\section{d. Relationship between bulk variables}

The $Z-R$ relationship is by far the most studied relationship between rainfall bulk variables in literature because of the problem of converting radar echoes into rainfall rates. In this case, using Eqs. (B4) and (B6) we obtain

$$
\begin{aligned}
Z= & {\left[\frac{1}{A_{m} T} \times\left(\frac{A_{m} T}{6 \pi 10^{-4}}\right)^{1.77 \overline{6}}\right] \times N^{-0.77 \overline{6}} } \\
& \times\left\{\frac{\Gamma(6.33)}{3.78 \Gamma(4)} \eta_{v}(6)[\eta(3)]^{-1.77 \overline{6}}\right\} \times R^{1.77 \overline{6}} .
\end{aligned}
$$

In Eq. (B12), the first term between square brackets is an "instrumentation" factor, while the term in between the second curl brackets is a "form factor," which depends on the particular shape of $p(D)$, and the relation between the drop velocity and its diameter. The existence of a bona fide (genuine and not a fit of convenience) 
power-law $Z-R$ relationship implies an interaction between the number of drops $N$ and the form factor such that the product between the drop occurrence factor $N^{-0.77 \overline{6}}$ and the drop pdf factor is proportional to $R^{\beta}$ so that

$$
Z \propto R^{1.77 \overline{6}+\beta} .
$$

An example of such interaction is the Marshall-Palmer distribution (Marshall and Palmer 1948), where the functional form of the volume diameter distribution $f(D)$ is

$$
\mathscr{N}(D)=N_{V} \frac{1}{\bar{D}} \exp \left(-\frac{D}{\bar{D}}\right)
$$

and

$$
N_{V} / \bar{D}=N_{0}=8000 \mathrm{~mm}^{-1} \mathrm{~m}^{-3} .
$$

In this case, using the exponential shape for the drop size distribution and the power-law relation Eq. (B2) for the drop velocity, we can write the relationships of Eq. (B4) in terms of $f(D)$ as

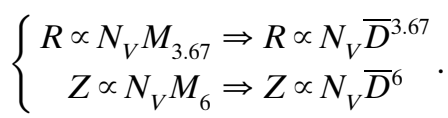

Using Eq. (B15) on both the above equation it follows

$$
Z \propto R^{7 / 4.67} \Rightarrow Z \propto R^{1.4989},
$$

which is very close to $Z \propto R^{1.47}$ fit reported by Marshall and Palmer (1948).

\section{e. Relationship between per unit drop bulk variables}

To "isolate" the contribution to the variability of the $Z-R$ relationship due to the particular shape of the flux diameter distribution $p(D)$, we adopt the "per unit drop" rainfall rate, and reflectivity. Namely, we divide $R$ by $N$ (the number of drops passing through the sampling area of the disdrometer in a time $T$ ), and $Z$ by $N_{V}$ (the concentration per unit volume) to obtain the "rescaled" variables $r$ and $z$. This transformation of the variables is along the same line of thinking proposed by Testud et al. (2001), who introduced normalized variables to investigate the normalized distribution of rain drops. In our case, the expression for $z$ and $r$ are

$$
\left\{\begin{array}{l}
r=\frac{R}{N}=\frac{6 \pi 10^{-4}}{A_{m} T} \eta(3) \Gamma(4) \mu^{3} \\
z=\frac{Z}{N_{V}}=\frac{\eta_{v}(6) \Gamma(6.33)}{\eta_{v}(0) \Gamma(0.33)} \mu^{6}
\end{array} .\right.
$$

To derive Eq. (B18), we start from Eq. (B4) and use Eq. (B6).

Finally, using Eq. (B18), we get the following relation for the reflectivity and rainfall rate per unit drop:

$$
z=C \zeta_{p} r^{2}
$$

where

$$
C=\left(\frac{A_{m} T}{6 \pi 10^{-4}}\right)^{2}
$$

is a constant "instrumentation" factor, and

$$
\zeta_{p}=\left\{\frac{\Gamma(6.33)}{\Gamma(0.33)[\Gamma(4)]^{2}}\right\} \frac{\eta_{v}(6)}{\eta_{v}(0)[\eta(3)]^{2}}
$$

is a shape factor that depends only on the particular shape of the flux diameter distribution $p(D)$, and the relation between velocity and drop diameter $v(D)$. If $p(D)$ is exponential then $\eta(3)=1$, and the shape factor is proportional to $\eta_{v}(6) / \eta_{v}(0)$ : a "deformation" due to the departure of the drop velocity from the formula of Eq. (B2). If $p(D)$ is exponential, and Eq. (B2) holds then also $\eta_{v}(6) / \eta_{v}(0)=1$, and the shape factor of Eq. (B21) is just a constant. Finally, if $v(D)$ is the power-law relation of Eq. (B2) but $p(D)$ is not exponential then via Eq. (B5), we get

$$
\zeta_{p}=\left\{\frac{\Gamma(6.33)}{\Gamma(0.33)[\Gamma(4)]^{2}}\right\} \frac{\eta(5.33)}{\eta(-0.67)[\eta(3)]^{2}} .
$$

In practice, to evaluate the shape factor $\zeta_{p}$, we use (depending on the type of disdrometer data and/or functional form for the rain drop velocity) Eqs. (B7), (B8), (B9), and (B11) to estimate $\left[M_{\alpha}, M_{\alpha, v}\right]$. We then estimate $(Z, R, z, r)$ and solve Eq. (B19) for $\zeta_{p}$.

All numerical computations reported in the present manuscript have been done using the Python package "disdrorain" publicly available at https:/github.com/ mignaccolo/disdrorain.

\section{REFERENCES}

Atlas, A., Ed., 1990: Radar in Meteorology. American Meteorological Society, $806 \mathrm{pp}$.

Atlas, D., and C. Chmela, 1957: Physical-synoptic variations of raindrop size parameters. Proc. Sixth Weather Radar Conf., Cambridge, MA, Amer. Meteor. Soc., 21-29.

_ , and C. W. Ulbrich, 1977: Path- and area-integrated rainfall measurement by microwave attenuation in the $1-3 \mathrm{~cm}$ band. J. Appl. Meteor., 16, 1322-1331, https://doi.org/10.1175/15200450(1977)016<1322:PAAIRM > 2.0.CO;2.

- R. C. Srivastava, and R. S. Sekhon, 1973: Doppler radar characteristics of precipitation at vertical indicence. Rev Geophys., 11, 1-35, https://doi.org/10.1029/rg011i001p00001. 
_ C. W. Ulbrich, F. D. Marks Jr., E. Amitai, and C. R. Williams, 1999: Systematic variation of drop size and radar-rainfall relations. J. Geophys. Res., 104, 6155-6169, https://doi.org/ 10.1029/1998JD200098.

Battan, L. J., 1973: Radar Observations of the Atmosphere. University of Chicago Press, $324 \mathrm{pp}$.

Cugerone, K., and C. De Michele, 2015: Johnson SB as general functional form for raindrop size distribution. Water Resour. Res., 51, 6276-6289, https://doi.org/10.1002/2014WR016484.

D'Adderio, L., K. Cugerone, F. Porcù, C. De Michele, and A. Tokay, 2016: Capabilities of the Johnson SB distribution in estimating rain variables. Adv. Water Resour., 97, 241-250, https://doi.org/10.1016/j.advwatres.2016.09.017.

Frasson, R., L. da Cunha, and W. Krajewski, 2011: Assessment of the thies optical disdrometer performance. Atmos. Res., 101, 237-255, https://doi.org/10.1016/j.atmosres.2011.02.014.

Ignaccolo, M., and C. De Michele, 2010: Statistical collapse of stratiform and convective drop diameter distributions at the ground. Geophys. Res. Lett., 37, L24402, https://doi.org/ 10.1029/2010GL045454.

_ instantaneous renormalized drop diameter distributions-Part 1: Convective vs. stratiform precipitation. Hydrol. Earth Syst. Sci., 16, 319-327, https://doi.org/10.5194/hess-16-319-2012.

$\longrightarrow$, and — 2012b: Skewness as measure of the invariance of instantaneous renormalized drop diameter distributions-Part 2: Orographic precipitation. Hydrol. Earth Syst. Sci., 16, 329-343, https://doi.org/10.5194/hess-16-329-2012.

— , and _ 2014: Phase space parameterization of rain: The inadequacy of gamma distribution. J. Appl. Meteor. Climatol., 53, 548-562, https://doi.org/10.1175/JAMC-D-13-050.1.

,$- \ldots$, and S. Bianco, 2009: The drop-like nature of rain and its invariant statistical properties. J. Hydrometeor., 10, 79-95, https://doi.org/10.1175/2008JHM975.1.

Jameson, A. R., and A. B. Kostinski, 2001: Reconsideration of the physical and empirical origins of Z-R relations in radar meteorology. Quart. J. Roy. Meteor. Soc., 127, 517-538, https:// doi.org/10.1002/qj.49712757214.

—_, and - 2002: Spurious power-law relations among rainfall and radar parameters. Quart. J. Roy. Meteor. Soc., 128 , 2045-2058, https://doi.org/10.1256/003590002320603520.

Kliche, D. V., P. L. Smith, and R. W. Johnson, 2008: L-moment estimators as applied to gamma drop size distributions. J. Appl. Meteor. Climatol., 47, 3117-3130, https://doi.org/10.1175/2008JAMC1936.1.

Kruger, A., and W. Krajewski, 2002: Two-dimensional video disdrometer: A description. J. Atmos. Oceanic Technol., 19, 602-617, https://doi.org/10.1175/1520-0426(2002)019<0602: TDVDAD $>2.0 . \mathrm{CO} ; 2$.

Licznar, P., and W. Krajewski, 2016: Precipitation type specific radar reflectivity-rain rate relationships for Warsaw, Poland. Acta Geophys., 64, 1840-1857, https://doi.org/10.1515/acgeo2016-0071.

List, R., 1988: A linear radar reflectivity-rainrate relationship for steady tropical rain. J. Atmos. Sci., 45, 3564-3572, https://doi.org/ 10.1175/1520-0469(1988)045<3564:ALRRRF > 2.0.CO;2.

Marshall, J. S., and W. M. Palmer, 1948: The distribution of raindrops with size. J. Meteor., 5, 165-166, https://doi.org/10.1175/ 1520-0469(1948)005<0165:TDORWS >2.0.CO;2.

_ , W. Hitschfeld, and K. L. S. Gunn, 1955: Advances in radar weather. Advances in Geophysics, Vol. 2, Academic Press, 1-56, https://doi.org/10.1016/S0065-2687(08)60310-6.

Martner, B. E., S. E. Yuter, B. W. Allen, S. Y. Matrosov, D. E. Kingsmill, and F. M. Ralph, 2008: Raindrop size distributions and rain characteristics in California coastal rainfall for periods with and without a radar bright band. J. Hydrometeor., 9, 408-425, https://doi.org/10.1175/2007JHM924.1.

Marzuki, M., W. Randeu, M. Schonhuber, V. Bringi, T. Kozu, and T. Shimomai, 2010: Raindrop size distribution parameters of distrometer data with different bin sizes. IEEE Trans. Geosci. Remote Sens., 48, 3075-3080, https://doi.org/ 10.1109/TGRS.2010.2043955.

Ochou, A. D., E. P. Zahiri, B. Bamba, and M. Koffi, 2011: Understanding the variability of $\mathrm{Z}-\mathrm{R}$ relationships caused by natural variations in raindrop size distributions (DSD): Implication of drop size and number. Atmos. Climate Sci., 1, 147-164, https://doi.org/10.4236/acs.2011.13017.

Orellana-Alvear, J., R. Celleri, R. Rollenbeck, and J. Bendix, 2017: Analysis of rain types and their Z-R relationships at different locations in the high Andes of southern Ecuador. J. Appl. Meteor. Climatol., 56, 3065-3080, https://doi.org/10.1175/JAMC-D-17-0009.1.

Raghavan, S., 2013: Radar Meteorology. Springer, 549 pp.

Rauber, R. M., and S. W. Nesbitt, 2018: Radar Meteorology: A First Course. Wiley, 488 pp.

Rosenfeld, D., and C. W. Ulbrich, 2003: Cloud microphysical properties, processes, and rainfall estimation opportunities. Radar and Atmospheric Science: A Collection of Essays in Honor of David Atlas, Meteor. Monogr., No. 52, Amer. Meteor. Soc., 237-258.

Steiner, M., J. A. Smith, and R. Uijlenhoet, 2004: A microphysical interpretation of radar reflectivity-rain rate relationships. J. Atmos. Sci., 61, 1114-1131, https://doi.org/10.1175/15200469(2004)061<1114:AMIORR > 2.0.CO;2.

Stout, G. E., and E. A. Mueller, 1968: Survey of relationships between rainfall rate and radar reflectivity in the measurement of precipitation. J. Appl. Meteor., 7, 465-474, https://doi.org/ 10.1175/1520-0450(1968)007<0465:SORBRR > 2.0.CO;2.

Testud, J., S. Oury, R. Black, P. Amayenc, and X. Dou, 2001: The concept of "normalized" distribution to describe raindrop spectra: A tool for cloud physics and cloud remote sensing. J. Appl. Meteor., 40, 1118-1140, https://doi.org/10.1175/15200450(2001)040<1118:TCONDT>2.0.CO;2.

Thorndahl, S., T. Einfalt, P. Willems, J. Nielsen, M. ten Veldhuis, K. Arnbjerg-Nielsen, M. Rasmussen, and P. Molnar, 2017: Weather radar rainfall data in urban hydrology. Hydrol. Earth Syst. Sci., 21, 1359-1380, https://doi.org/10.5194/hess-21-1359-2017.

Tokay, A., and D. A. Short, 1996: Evidence from tropical raindrop spectra of the origin of rain from stratiform versus convective clouds. J. Appl. Meteor., 35, 355-371, https://doi.org/10.1175/ 1520-0450(1996)035<0355:EFTRSO > 2.0.CO;2.

—, A. Kruger, and W. Krajewski, 2001: Comparison of drop size distribution measurements by impact and optical disdrometers. J. Appl. Meteor., 40, 2083-2097, https://doi.org/10.1175/15200450(2001)040<2083:CODSDM>2.0.CO;2.

Uijlenhoet, R., 2001: Raindrop size distributions and radar reflectivityrain rate relationships for radar hydrology. Hydrol. Earth Syst. Sci., 5, 615-627, https://doi.org/10.5194/hess-5-615-2001.

Ulbrich, C., 1983: Natural variations in the analytical form of the raindrop size distribution. J. Climate Appl. Meteor., 22, 1764-1775, https:// doi.org/10.1175/1520-0450(1983)022<1764:NVITAF>2.0.CO;2.

Williams, C. R., 2009: Accuracy of reflectivity estimated from profiling radars. 2009 IEEE Radar Conf., Pasadena, CA, IEEE, 1-4, https://doi.org/10.1109/RADAR.2009.4976996.

Yuter, S. E., and R. A. Houze, 1997: Measurements of raindrop size distributions over the Pacific warm pool and implications for $Z-R$ relations. J. Appl. Meteor., 36, 847-867, https://doi.org/ 10.1175/1520-0450(1997)036<0847:MORSDO>2.0.CO;2. 